\title{
Computations of AC Loss in the ITER Magnets During Fast Field Transients
}

\author{
Luca Bottura, Pierluigi Bruzzone, Jonathan B. Lister, Claudio Marinucci, and Alfredo Portone
}

\begin{abstract}
The calculation of AC loss due to the control currents in ITER is a cumbersome task. The reason is that control transients require small field changes $(0.1 \mathrm{~T}$ or less) at moderate frequency (up to $10 \mathrm{~Hz}$ ), where effects of partial penetration of the filaments and shielding are important and need to be taken into account to produce sound AC loss estimates. In this paper we describe models developed for AC loss calculation, in particular hysteresis and coupling current loss, that are suitable for the above regime. Both hysteresis and coupling loss models are adapted to the conductor analysed through few parameters (the effective filament diameter and time constants) that can be derived from measurement of loss on short samples. We report an example of calculations of AC loss in the ITER TF and PF coils for two vertical control scenarios (VS1 and VS2) during high beta operation at flattop.
\end{abstract}

Index Terms-AC loss, coupling currents, hysteresis, pulsed superconducting magnets.

\section{INTRODUCTION}

A C LOSS in superconducting magnets is usually dominated by two contributions that originate within the superconducting strands and cables:

- hysteresis loss in the superconducting filaments;

- coupling loss within strands and among strands in a cable or composite.

The first component, hysteresis loss, is caused by persistent currents induced within the filament by field changes. Persistent currents produce a magnetization of hysteretic nature. Hysteresis loss involves thus the superconducting filaments only. The second component, coupling loss, is originated by electromagnetic coupling among filaments in a strand, and among strands in a cable. Coupling currents flow partially in the superconductor, partially in resistive contacts among them, and they dissipate power in the resistive transition. Coupling loss thus involve the cable as a whole unit. The next sections deal with each component separately, proposing a flexible calculation algorithm to cope with most practical situations in a superconducting magnet.

Manuscript received August 28, 2006.

L. Bottura is with CERN, Geneva, CH-1211 Switzerland (e-mail: Luca.Bottura@cern.ch).

P. Bruzzone and C. Marinucci are with EPFL-CRPP, Fusion Technology Division, CH-5232 Villigen PSI, Switzerland.

J. B. Lister is with EPFL-CRPP, CH-1015 Lausanne Switzerland.

A. Portone is with EFDA-CSU, D-85748 Garching, Germany.

Color versions of one or more of the figures in this paper are available online at http://ieeexplore.iee.org.

Digital Object Identifier 10.1109/TASC.2007.899837

\section{HySTERESIS LOSS CALCULATION}

The calculation of hysteresis loss in a superconducting filament can be a complex task, especially when the magnetic field variation is arbitrary. The calculation method proposed below is based on tracking the magnetic and electric field profiles inside the filament. This allows, at each time, to compute the instantaneous, local heat density given by the product of electric field and current density. The average power and the total energy dissipation in the superconductor are then obtained by integrals in space and time of the local heating power density. As we wish to achieve reliable and fast calculation, we obviously aim at having analytic solutions for the field profiles inside the superconducting filament, which is a non-trivial task. Here we follow an approximate approach, based on the following assumptions:

- the filaments are round, and are not coupled;

- the change of the magnetic field components in each space direction $k(k=1 \ldots 3)$ is treated separately, that is the effect of variation of each component is considered as independent from the variation of the other two components. The only coupling between field components arises through the value of the critical current density, which depends on the field module;

- the critical current density is uniform in the filament cross section;

- transport current effects are neglected.

Thanks to these simplifying assumptions, the magnetic and electric field profiles inside the filament can be computed in closed form for a cylindrical filament in parallel field [1]. In the case of a cylindrical filament in transverse field, however, only approximations are available [2], [3]. Therefore, in addition to the assumptions above, we choose to approximate a cylindrical filament in a transverse field with a slab of suitably scaled thickness (see later for the scaling), for which a closed form solution of the field profiles is available. In the sections below we report the expressions strictly necessary for the loss calculation in the case of an arbitrary field change. Throughout, we use the normalized quantities as defined in Table I. Note that the variable $x$ spans the slab thickness or the cylinder radius, while the indexes of field and current density components are not indicated as all vectors have a single component, $z$ for the magnetic field and $y$ for the current density and electric field.

\section{A. Slab Solution}

The field profile in a superconducting slab subjected to an external field change is piecewise linear, starting from the external value $h_{e}$ at $x=1$ (the slab boundary in normalized coor- 
TABLE I

NORMALIZATION FOR HYSTERESIS LOSS CALCULATION

\begin{tabular}{ll}
\hline effective filament diameter & $D$ \\
normalized space co-ordinate & $x=X / \mathrm{D} / 2$ \\
critical current density at zero field & $J_{c 0}=J_{c}(0)$ \\
virgin penetration field & $H_{p 0}=J_{c 0} D / 2$ \\
normalized critical current density & $j=J_{c}(B) / J_{c 0}$ \\
normalized magnetic field & $h=H / H_{p 0}$ \\
normalized electric field & $e=E / \mu_{0} H_{p 0} D / 2$ \\
normalized power & $p=P / \mu_{0} H_{p 0}{ }^{2}$ \\
\hline
\end{tabular}

dinates). The outermost layer, being penetrated by the external field change, has a normalized field:

$$
h=h_{e} \pm j(1-x)
$$

where the sign of the current density on the right hand side in (1) is determined by the direction of the field change. The depth at which the field profile penetrates inside the slab depends on the state of the superconductor, and two cases are possible: a virgin portion of the slab (no previous shielding current layer), or a portion of the slab with frozen field (a previously established shielding layer). The normalized penetration depth $x_{p}$ in the two cases is:

$$
x_{p}=\left\{\begin{array}{ll}
1-\frac{\left|h_{e}\right|}{j} & \text { virgin } \\
1-\frac{\left|h_{e}\right|}{2 j} & \text { non virgin }
\end{array} .\right.
$$

The normalized electric field in the outermost layer, being penetrated (i.e. for $u_{p} \leq x \leq 1$ ) is given by:

$$
e=\frac{\partial h_{e}}{\partial t}\left(x-x_{p}\right)
$$

and is zero elsewhere. The local value of the dissipated power density is the product of the electric field (given by (3)), and the current density in the penetration layer. The average normalized power density in the slab is then:

$$
\bar{p}=\int_{x_{p}}^{1} \frac{\partial h_{e}}{\partial t} j\left(x-x_{p}\right) d x=\frac{\partial h_{e}}{\partial t} j \frac{\left(1-x_{p}\right)^{2}}{2} .
$$

The above expressions are sufficient to solve the general case of arbitrary variation of the external field, keeping track of the shielding layers and their appearance/disappearance as the external field changes. To this aim, the magnetic field changes are subdivided in time in piecewise linear portions. The information needed by the tracking process consists, for each of the linear field swings, in the penetration depth $x_{p}$ of a shielding current layer, the magnetic field $h_{e}$ that caused it, and the direction of the shielding currents.

\section{B. Scaling of the Slab Solution}

The solution presented in the previous section for a slab can be scaled to represent the penetration of a cylinder in transverse field. The scaling is done so that the asymptotic behaviors of the equivalent slab and cylinder are the same for small and large field changes. To this aim we use the following known expressions [2] for the energy lost per cycle and per unit volume $Q$ in the case of a slab in a parallel alternating field with total field swing $B_{m}$ (peak to peak amplitude of the field change):

$$
Q_{s}= \begin{cases}\frac{B_{m}^{2}}{2 \mu_{0}} \frac{\beta}{3} & \text { for } \beta \leq 1 \\ \frac{B_{m}^{2}}{2 \mu_{0}}\left(\frac{1}{\beta}-\frac{2}{3 \beta^{2}}\right) & \text { for } \beta>1\end{cases}
$$

and for a cylinder in the same transverse alternating field:

$$
Q_{c}=\left\{\begin{array}{ll}
\frac{B_{m}^{2}}{2 \mu_{0}} \frac{2}{3}\left(2 \beta-\beta^{2}\right) & \text { for } \beta \leq 1 \\
\frac{B_{m}^{2}}{2 \mu_{0}} \frac{2}{3}\left(\frac{2}{\beta}-\frac{1}{\beta^{2}}\right) & \text { for } \beta>1
\end{array} .\right.
$$

The parameter $\beta$ above is the ratio of the field swing to the penetration field $2 B_{p}$ :

$$
\beta=\frac{B_{m}}{2 B_{p}}
$$

where we recall that the (first) penetration field is given by:

$$
\begin{array}{ll}
\text { slab : } & B_{p}=\mu_{0} J_{c} \frac{D_{s}}{2} \\
\text { cylinder : } & B_{p}=\mu_{0} J_{c} \frac{D_{c}}{\pi}
\end{array}
$$

with $D_{s}$ and $D_{c}$ respectively slab thickness and cylinder diameter. Inspecting (5) and (6), we can obtain the same dissipated energy per cycle in the limits $\beta \rightarrow 0$, and $\beta \rightarrow \infty$ if we use a slab effective thickness $D_{s}=(8 / 3 \pi F) D_{c}$, and scale the energy per cycle by a factor $F=2.309$.

\section{Cylinder in Parallel Field}

A cylinder in parallel field is described by equations that are very similar to those of a slab, treated previously. The magnetic field profile is indeed the same as in the case of the slab, so that (1) and (2) hold in both cases. The electric field contains terms that are originated from the rot differential operator in cylindrical symmetry, and in the outermost layer, being penetrated, it is given by:

$$
e=-\frac{1}{2} \frac{\partial h_{e}}{\partial t} \frac{x^{2}-x_{p}^{2}}{x}
$$

From (10) we compute the average power density in the cylinder:

$$
\bar{p}=\frac{1}{\pi} \int_{x_{p}}^{1} \frac{j}{2} \frac{\partial h_{e}}{\partial t} \frac{x^{2}-x_{p}^{2}}{x} 2 \pi x \quad d x=\frac{j}{3} \frac{\partial h_{e}}{\partial t}\left(1-3 x_{p}^{2}+2 x_{p}^{3}\right)
$$

\section{COUPLing Loss CALCUlation}

The calculation of coupling currents in the complex cabling geometry of a large size Cable-in-Conduit Conductor (CICC) can be just as daunting as an exact calculation of hysteresis in an arbitrary filament. Here, also, we make simplifying assumptions:

- the cable can be described macroscopically by three time constants $/_{k}$ and three demagnetization shape factors $n_{k}$ [2]. Each time constant and demagnetization factor $/ k$ and $n_{k}$ refer to a space direction $k$ in the cable; 
- as for hysteresis loss, we consider the three cable directions as completely independent, and solve for each direction independently from the other;

- the cable is not saturated, and coupling currents can flow unperturbed in the cable.

We stress that we use the same algorithm for field changes in all directions, and we do not treat the loss due to field changes along the axis of the cable (parallel loss) separately. The reason is that there is no recent experimental evidence that parallel field loss in a CICC has a significant impact.

The first step in the calculation of the coupling current loss is the integration of the equation governing the internal field in the cable [1]:

$$
\frac{\partial B_{i}}{\partial t}+\frac{B_{i}}{\tau}=\frac{B_{e}}{\tau}
$$

where $B_{i}$ is the field in the composite and $B_{e}$ is the external, changing field. Note that, as we treat the three space direction in the same way, we drop indices from here on. To obtain an analytical solution, we assume that the external field changes piecewise linearly in time. During each time interval we can hence write that $B_{e}=B_{e}^{0}+B_{e}^{1} t$. If we indicate with $B_{i}^{0}$ the initial value of the internal field at the beginning of the time interval considered, we can solve Eq. (3.1), leading to the following integral:

$$
B_{i}=B_{e}^{0}+B_{e}^{1}(t-\tau)+\left[B_{i}^{0}-\left(B_{e}^{0}-B_{e}^{1} \tau\right)\right] e^{-\frac{t}{\tau}}
$$

The last term in (13) is a decaying exponential with time constant / that describes the shielding phase for fast field changes. Once the exponential has decayed, the contribution of the third term is negligible, and the internal field is equal to the external field delayed by $/$.

Equation (13) provides the evolution of the field internal to the composite, once the initial condition is known (see later), and can be derived to give the internal field change rate:

$$
\frac{\partial B_{i}}{\partial t}=B_{e}^{1}-\frac{\left[B_{i}^{0}-\left(B_{e}^{0}-B_{e}^{1} \tau\right)\right]}{\tau} e^{-\frac{t}{\tau}} .
$$

This is the desired result, used to calculate the instantaneous power dissipated as:

$$
P=\frac{n \tau}{\mu_{0}}\left(\frac{\partial B_{i}}{\partial t}\right)^{2}
$$

and finally the energy during a time interval (generically indicated below as $[0 \ldots T]$ ) with linear field swing:

$$
\begin{aligned}
E= & \int_{0}^{T} P d t \\
= & \frac{n \tau}{\mu_{0}} B_{e}^{1^{2}} T-\frac{n}{2 \mu_{0}}\left[B_{i}^{0}-\left(B_{e}^{0}-B_{e}^{1} \tau\right)\right]^{2}\left(e^{-2 \frac{T}{\tau}}-1\right) \\
& +\frac{2 n \tau}{\mu_{0}} B_{e}^{1}\left[B_{i}^{0}-\left(B_{e}^{0}-B_{e}^{1} \tau\right)\right]\left(e^{-\frac{T}{\tau}}-1\right) .
\end{aligned}
$$

The coupling loss calculation algorithm uses the above equations for each field direction $k$, in turn. The internal field during

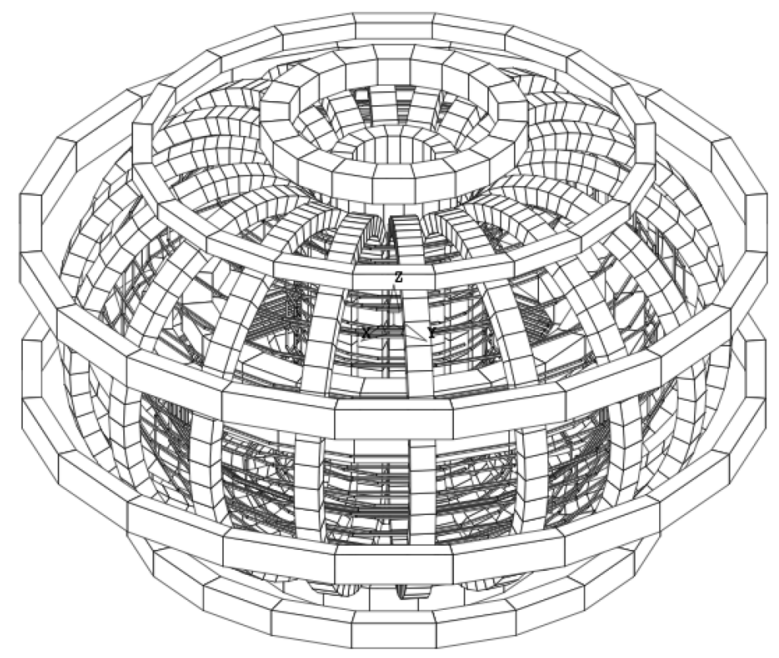

Fig. 1. Model of the coil geometry of the ITER coils for the study of AC loss in vertical stabilization scenarios.

a time interval with a linear field swing is tracked using (13), which gives in particular the value at the end of the swing to be used for the following time interval. Equations (15) and (16) are then used to compute instantaneous power and energy dissipated during the field swing. Following this logic, the calculation must keep track, for each cable, of the internal field at the end of the swing, that is used as initial condition for the following swing.

\section{EXAMPLE OF APPLICATION}

As an example of the use of the above algorithms, we report the results of calculations of AC loss in the ITER TF and PF coils for two vertical plasma stabilization scenarios (VS1 and VS2) during high beta operation at start of burn (SOB). The magnetic model built to this aim includes all CS, PF and TF coils, as well as some 60 axisymmetric passive circuits that represent the conductive wall of the vacuum vessel. A 3-D rendering of the model is shown in Fig. 1. The coil and conductor data have been taken from the reference design reported in [5]. In particular, for loss calculations, the effective filament diameter of $\mathrm{Nb}_{3} \mathrm{Sn}$ is $30 \mu \mathrm{m}$, while for NbTi it is $6 \mu \mathrm{m}$. The coupling loss time constant is $25 \mathrm{~ms}$ in all space directions for all conductors. The current variation during the vertical control scenarios has a wide frequency spectrum (typically up to $10 \mathrm{~Hz}$ ), and produces field changes of relatively small amplitudes (few 10's of mT on the $\mathrm{PF}$ coils, up to $0.1 \mathrm{~T}$ in the $\mathrm{CS}$ coils).

The AC loss calculation requires the knowledge of the three magnetic field components at each conductor location in the coil winding. This calculation has been done using standard linear magnetostatic techniques.

We report in Fig. 2 a summary of the overall results of these two simulations. The bars represent the total loss, split among each of the three main coil systems, and further subdivided in the different mechanism (coupling and hysteresis). Loads are reported as average power during the time simulated, $10 \mathrm{~s}$. The calculation provides a quick means to qualify the controller scenarios in terms of the cryogenic load, as we see a clear distinction in the loss at the level of the CS and PF system. We note further that the contribution of the two loss mechanisms in the 

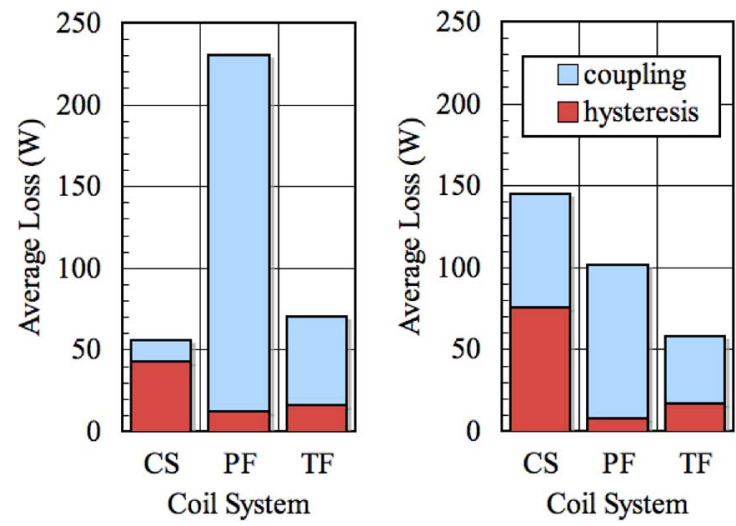

Fig. 2. Average AC loss power computed for the vertical stabilization scenarios VS1 (left) and VS2 (right) at SOB.

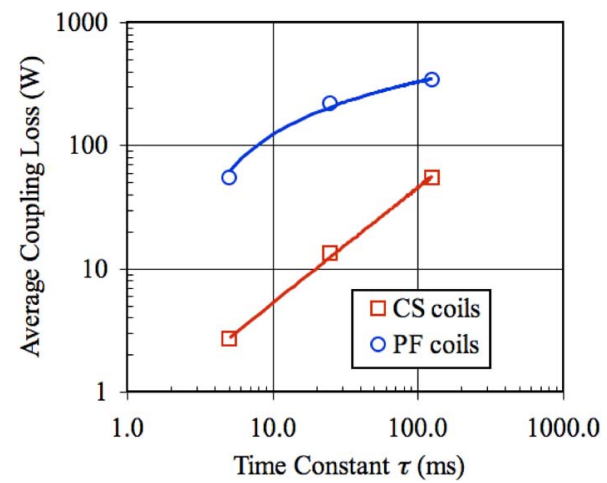

Fig. 3. Sensitivity study: effect of time constant on coupling current loss in the CS and PF coil systems during the VS1 scenario.

CS and PF coil systems is massively different in the two scenarios. This is due to the combined effect of the different current amplitudes as well as the different dynamic characteristics of the current waveforms in the CS and PF coils, affecting both hysteresis and coupling loss. Interestingly enough, the situation on the TF coil (close to the plasma) is essentially unaffected by the control scenario, as we should have expected.

To complete our example, we have performed sensitivity studies on the effect of a change in the loss parameters of the conductors. Fig. 3 shows in particular the effect of a parametric change of $\tau$ by a factor $1 / 5$ to 5 (i.e. from $5 \mathrm{~ms}$ to $125 \mathrm{~ms}$ ) on the coupling loss in scenario VS1. As a side remark, this range of variation is representative for the spread measured on the large-scale ITER cables. The scaling of the coupling loss in the CS coil system is approximately linear, which indicates negligible shielding in the range of time constants explored for the specific scenario analysed (low frequency regime). In the PF coil system, on the other hand, we clearly see the effect of shielding at high values of $\tau$, which results in a coupling loss significantly smaller than would be expected by the low-frequency regime, linear extrapolation.

\section{CONCLUSION}

We have presented a calculation method for AC loss in pulsed superconducting magnets that is suitable over a wide regime of field changes (from partial to full penetration) and frequencies (from the low frequency limit to shielding). The example reported, vertical control scenarios in ITER, provides a measure of the flexibility in dealing with complex geometric and powering conditions. The model can be useful for other applications of similar nature, e.g. pulsed accelerator magnets requiring loss optimization.

\section{ACKNOWLEDGMENT}

The authors gratefully acknowledge the crucial contribution of Dr. C. Rosso (CryoSoft, France) in producing the solution of the coupling current loss. The slab-cylinder scaling is based on an idea of Dr. J. Minervini (MIT, USA).

\section{REFERENCES}

[1] H. Brechna, Superconducting Magnet Systems. : Springer-Verlag, 1973.

[2] M. Wilson, Superconducting Magnets. Oxford: Clarendon Press, 1983.

[3] W. J. Carr, Jr., AC Loss and Macroscopic Theory of Superconductors. : Gordon and Breach Science Publishers, 1983.

[4] A. M. Campbell, "A general treatment of losses in multifilamentary superconductors," Cryogenics, vol. 22, no. 1, pp. 3-16, 1982.

[5] ITER Design Description Document. Magnet Section 1: Engineering description, N11 DDD 178 04-06-04 R 0.4, 2004. 